九州大学学術情報リポジトリ

Kyushu University Institutional Repository

\title{
EXTEND LECTURES FOR KIDS ABOUT INFORMATION INTO SAMPLING THEOREM AND THE FOURIER TRANSFORM
}

Ikeda, Daisuke

Faculty of Information Science and Electrical Engineering, Kyushu University

http://hdl. handle. net/2324/4495646

出版情報：INTED2019 Proceedings，pp.7419-1079，2019-03-11. IATED バージョン：

権利関係 : 


\title{
EXTEND LECTURES FOR KIDS ABOUT INFORMATION INTO SAMPLING THEOREM AND THE FOURIER TRANSFORM
}

\author{
D. Ikeda \\ Kyushu University (JAPAN)
}

\begin{abstract}
Computer science education for kids has received considerable attention. However, we can hardly find educational cases for kids in information science, compared to computational science, such as programming. Aiming to develop classes and their materials about information science for kids, the author has tried to develop lectures to deliver core ideas of the Fourier transform, which is an important topic of information science, using the notion of average. However, these lectures did not include the sampling theorem, which plays an important role when we decide the sampling rate when digitizing analog data, and thus it is essential in information science. One of the challenges to incorporate it to lessons for kids is that it is necessary to explain notions of the wave and its frequency while lectures about the Fourier transform itself do not require these notions since the author explains the main idea of it using average. To incorporate the sampling theorem into our lectures, the author tried to explain waves and frequencies by timing. In this paper, the author shares experiences extending already developed lectures so that they include the sampling theorem. One important feature of these lessons is that we have used many hands-on materials. For the sampling theorem, we also use an equipment, called "water pearl", which makes us confuse that droplets of dropping water seem to be climbing up when we see it through flashing light. This is useful when we learn basic ideas of the sampling theorem because this phenomenon happens when two frequencies of dropping and flashing are appropriately selected. In this paper, the author also explain how we use this equipment to explain the sampling theorem.

Keywords: Information Theory, Fourier Transform, Sampling Theorem, Average, Vector Space, Inner Product.
\end{abstract}

\section{INTRODUCTION}

In recent years, computer science education for kids has received considerable attention. We can see many programming languages, whose main targets are kids, such as Scratch $\mathrm{Jr}$ (https://www.scratchjr.org/). In addition to programming languages, materials to teach computer science for kids without using computers have been developed, such as $[1,2]$ and CS Fundamentals Unplugged (https://code.org/curriculum/unplugged). These materials are designed to deliver essential ideas of fundamental theories of computer science. Since these languages and materials enable experience-based learning, kids can enjoy learning with a trial and error process.

The recent attention mainly focuses on topics in computational science, but not on those in information science, where we consider that computer science has followings as its sub-fields: computational science, informational science, and artificial intelligence. The author thinks that this is partly because there do not many hands-on materials about information science and thus it is difficult for teachers to teach kids topics in information 
science. In case of programming, which is a typical topic of computational science, teachers can use many visual tools for kids. Even in lectures of information science for kids, only some basic ideas in it are treated, such as binary representation and error detection, and more complex themes, such as frequency representation, are not included. Our goal to this direction is to develop lectures and related materials about information science for kids. To do that, the author has already tried to develop lectures to deliver core ideas of the Fourier transform, which is an important topic of information science and relates with frequency representation. The author has used the notion of average, which is learned by elementary school students and so they can understand the lectures. To support the lectures, the author has also developed a JavaScript application (https://rebrand.ly/486fe), which was already presented at this conference.

However, these lectures did not include the sampling theorem, which plays an important role when we decide the sampling rate for digitizing analog data, and thus it is essential in information science. One of the challenges to incorporate it to lessons for kids is that it is necessary to explain notions of the wave and its frequency while lectures about the Fourier transform already developed do not require these notions since the author explains the main idea of it using average.

In this paper, the author shares experiences extending already developed lectures into those including the sampling theorem, using the notion of timing instead of waves and frequencies. One important feature of these lessons is that we have used many hands-on materials. For the sampling theorem, we use an equipment, called "water pearl", which makes us confuse that droplets of dropping water seem to be climbing up when we see it through flashing light. Thanks to this mysterious phenomenon, kids love to see it. In addition to that, this is useful when we learn basic ideas of the sampling theorem because this phenomenon happens when two frequencies of dropping and flashing are appropriately selected.

\section{BASIC NOTIONS OF INFORMATION SCIENCE}

In this section, we will explain some basic notions of information science, including sampling and digitization, without going into detailed mathematical notions.

\subsection{Digitization}

Information science basically shows how to express data, functions, or events in digital format. Here we use only the term "data" as the representative for them. In information science, it is assumed that there exit two types of data: analog and digital data, and that real phenomena are represented as analog data. 


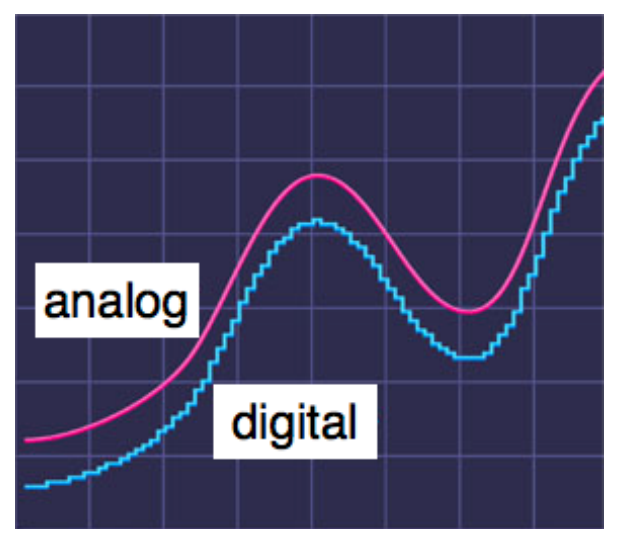

Figure 1. It is assumed in information science that a real world data is represented as analog data, which corresponds to a continuous line (red line). The blue line is a corresponding digital data.

One of the typical expressions for data is vector representation. The vector representation can be used for both analog and digital data, and we begin with digital one. To express digital data in Fig. 1 in vector representation, we use a sequence of numbers $\left(y_{1}, y_{2}, \cdots, y_{n}\right)$, each of which shows the height of a horizontal part of digital data from left to right, where it is assumed that corresponding $\left(x_{1}, x_{2}, \cdots, x_{n}\right)$ is fixed. In case of analog data, we have to use a sequence of infinitely many numbers since there exist infinitely many points even in the fixed range of $\left[x_{1}, x_{n}\right]$.

Digitizing is to convert analog data into digital one, with quantization and sampling. Roughly speaking quantization is to round a real number $y_{i}$ into a decimal number with some fixed digits after the decimal point, while sampling is chosen a finite number of $\left(x_{1}, x_{2}, \cdots, x_{n}\right)$ from $\left[x_{1}, x_{n}\right]$, which include infinitely many real numbers.

\subsection{Fourie Transform}

According to [3], we briefly explain the Fourier series, which is a specific version of the Fourier transform.

The Fourier series decomposes a function into simpler trigonometric functions. That is, any function $\mathrm{f}(\mathrm{x})$ can be decomposed in a Fourier series as follows:

$$
\mathrm{f}(\mathrm{x})=\frac{a_{0}}{2}+\sum_{k=1}^{\infty} a_{k} \cos k x+b_{k} \sin k x,(1)
$$

where $a_{0}, a_{k}, b_{k}$ are called Fourier coefficients and defined as follows:

$$
\begin{gathered}
a_{0}=\frac{1}{\pi} \int_{-\pi}^{\pi} f(x) d x, \\
a_{k}=\frac{1}{\pi} \int_{-\pi}^{\pi} f(x) \cos k x d x, \text { and } \\
b_{k}=\frac{1}{\pi} \int_{-\pi}^{\pi} f(x) \sin k x d x .
\end{gathered}
$$

Intuitively, these coefficients show amount of trigonometric functions included in the original $\mathrm{f}(\mathrm{x})$. Therefore, with the Fourier series, we can obtain the corresponding vector representation $\left(a_{0}, a_{1}, b_{1}, \cdots, a_{n}, b_{n}, \cdots\right)$, which contains infinitely many elements. To explain it, instead of using abstract notion of functions and vectors, we use the following block representation, as follows: 


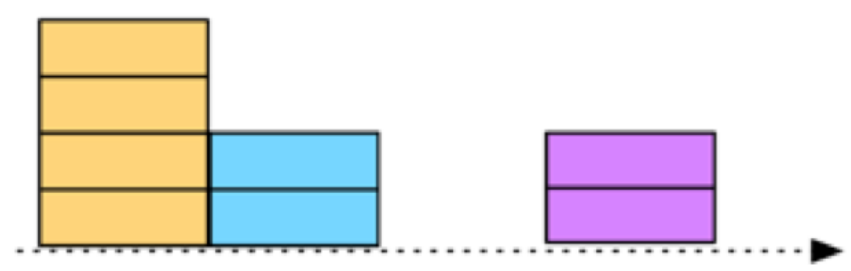

Figure 2. A block representation for (4, 2, 0, 2).

The shape of blocks corresponds to a vector in four dimensional space. The shape of Fig. 2 corresponds to $(4,2,0,2)$. If we use more blocks, we can represent vectors in a higher dimensional space, even in the infinite dimension.

Now we consider another representation in vector representation. In the case of Fig. 2, we try to find another representation, instead of $(4,2,0,2)$. First, given a block shape, we compute the average of four hights of blocks, like, $(4+2+2+0) / 4=2$. The average of all blocks corresponds to $a_{0}$. Using only the average, that is, using $(2,2,2,2)$, the obtained shape looks like the right-hand side of Fig. 3.

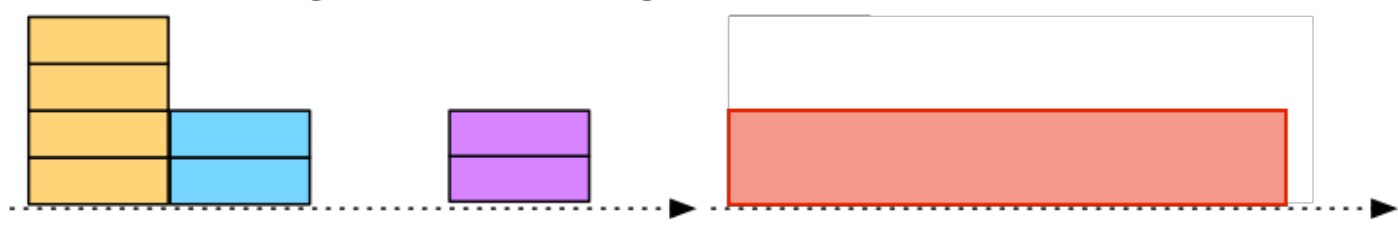

Figure 3. The original shape of blocks (the left-hand side) and $(2,2,2,2)$ showing the average of all rows, $(4,2,0,2)$ (the right-hand side)

Next, we compute a pair of two averages of the left-half $(4,2)$ and right-half $(0,2)$ of $(4,2$, 0,2 ), and obtain $(4+2) / 2=3$ and $(0+2) / 2=1$ as a result, respectively. Given 3 and 1 as two averages of the left and right halves, that is, $(3,3,1,1)$, we can sketch it the shape like blue line of the right-hand side of Fig. 4.

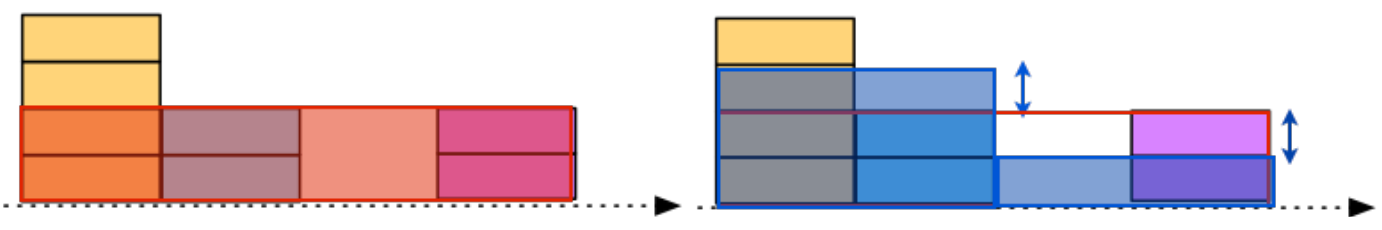

Figure 4. The average $(2,2,2,2)$ of $(4,2,0,2)$ (the left-hand side), and $(3,3,1,1)$ of two averages of two rows $(4,2)$ and the other rows $(0,2)$, respectively (the right-hand side)

Compared to the left-hand side of Fig. 4, (2, 2, 2, 2), we can grasp a more detailed shape from the right-hand side of Fig. 4, (3, 3, 1, 1).

In the Fourier series (and transform), we do not use two averages directly, but the total average 2 and the difference 1 between 2 and $(3,1)$, where 1 is obtained from the following fact that the absolute values of the following differences, $3-2=1$ and $1-2=-1$, are the same, assuming the left half is positive. We can rebuild the shape $(3,3,1,1)$ of two averages 3 and 1 of left and right halves from 2 and 1 by calculating $2+1$ and $2+(-1)$. This rebuild process is illustrated as blue arrows in Fig. 4.

Finally, we compute another pair of two averages: the average of two outer rows and one of two inner rows. In case of $(4,2,0,2)$, the average of two outer rows $(4,2)$ is 3 , and one of two inner rows $(2,0)$ is 1 . The difference of these two averages from the total average 2 is 1 , where we assume the tow outer rows are positive. Therefore, we can rebuild the 
original shape (the right-hand side of Fig. 5), given the total average 2, the difference 1 of left and right halves from the total average, and the difference 1 of outer and inner halves from the total average, by adding 1 to outer rows of the shape of the left-hand side and subtracting 1 from inner rows of the left-hand side of Fig. 5 (see blue arrows in Fig. 5).

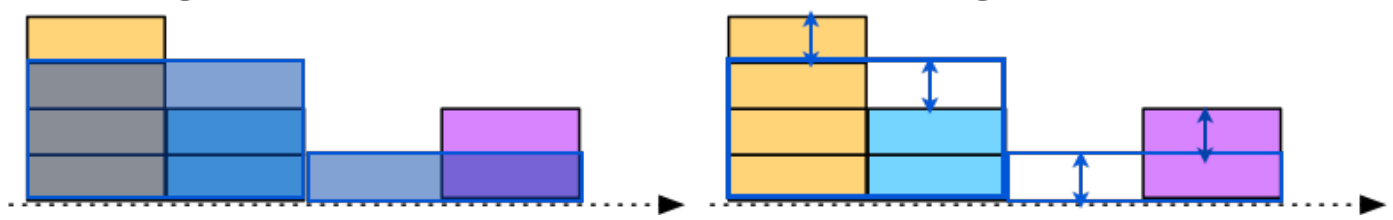

Figure 5. $(4,2,0,2)$ (the right-hand side) is obtained by adding 1 to two outer rows of $(3,3,1,1)$ (lefthand side) and subtracting 1 from two inner rows of it.

Although we can rebuild the original shape from three digits, the total average 2 and two differences 1 and 1 , in this case, four digits are necessary in general because the original shape corresponds to a vector in four dimensional space. In this sense, 0 is the final element for another representation, and finally we have $(2,1,1,0)$ from $(4,2,0,2)$, where, from left to right, 2 is the total average, 1 the difference of left and right halves, 1 the difference of outer and inner halves, and 0 the difference of 1 st, 3rd rows $(4,0)$ and 2 nd, 4 th rows $(2,2)$.

\section{SAMPLING THEOREM}

In the previous section, we have explained basic ideas of the Fourier transform, using various averages. Thanks to averages, we do not need to use waves and frequencies, which are essential notions of the Fourier transform. In fact, we can see trigonometric functions in Equation (1), where one trigonometric function is one wave with some frequency.

To understand the sampling theorem, we need to understand waves and frequencies of trigonometric functions. But now we use timing of vectors instead. As described above, we can represent a continuous function as a vector with infinitely many elements, and a trigonometric function is continuous.

In the previous section, we have used four types of averages. They correspond to the following four base vectors:

$$
\frac{1}{4}(1,1,1,1), \frac{1}{4}(1,1,-1,-1), \frac{1}{4}(1,-1,-1,1), \frac{1}{4}(1,-1,1,-1),(2)
$$

where the last three vectors have both positive and negative values. Moreover, elements in the 2 nd and the $3 r d$ vectors change from positive to negative or vice versa every two elements, while those in the 4th one change every element. These change timings correspond to frequencies, and the 4th vector has a higher frequency while the 2 nd and 3rd ones have a lower one.

We can see these changes from positive to negative in trigonometric functions. In graphs of Fig. 6 , there exists three timings, that is, frequencies, where $\sin (x)$ has the lowest frequency while $\cos (3 x)$ the highest one. 


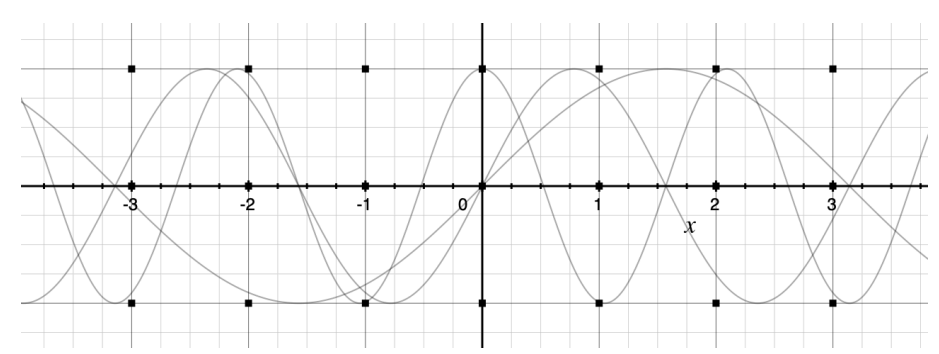

Figure 6. Three graphs of $\sin (x), \sin (2 x)$, and $\cos (3 x)$.

A vector with a fast timing (high frequency) describes sharp changes in data while one with a slow timing (low frequency) gradual changes in data. We can easily see such sharp and gradual changes in images in Fig. 7, where the original image is given at the left-hand side, a low-pass filtered image, that is, an image with only guradual changes, at the center, and a high-pass filtered one, that is, an image with only sharp changes, at the right-hand side. In a high-pass filtered image, there exist only boundaries between different objects since a boundary is a sharp change.
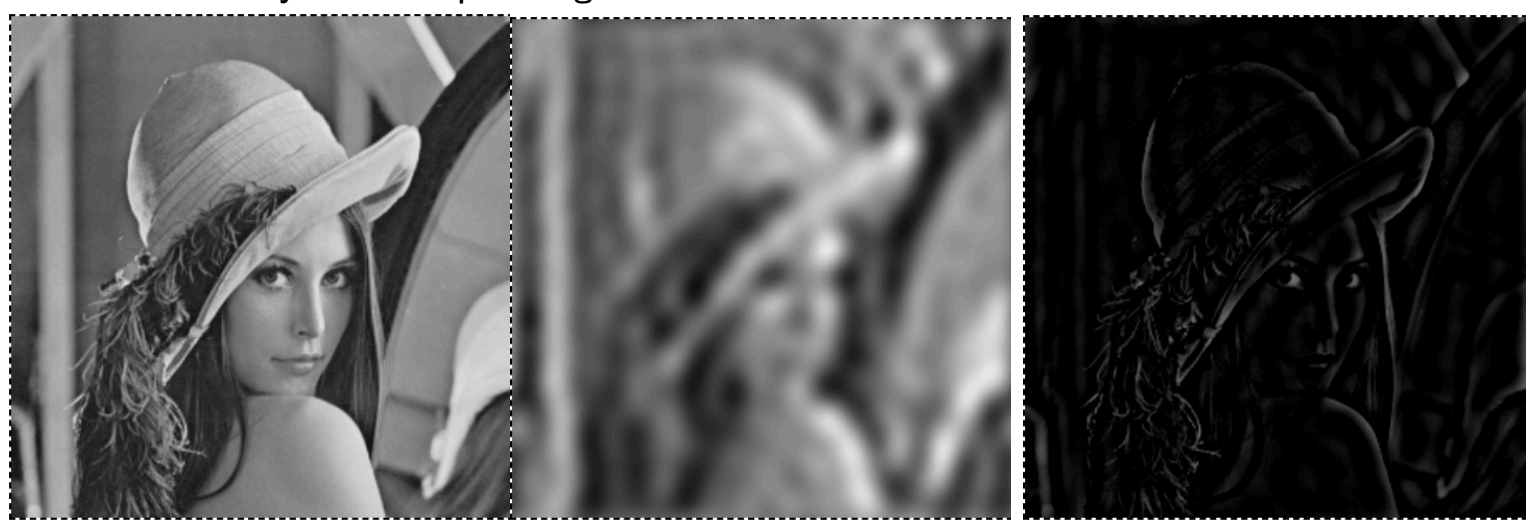

Figure 7. Original image (left), low-pass filtered image (center), and high-pass filtered image (right)

Using the vectors in (2), we can compute any of four averages described in the previous section, by calculating the inner product of the original shape (vector) and one of them. For example, as the inner product of $(4,2,0,2)$ with $1 / 4(1,1,-1,-1)$, we have the following:

$$
\frac{1}{4}(1,1,-1,-1) \cdot(4,2,0,2)=\frac{1}{4}(4+2+0-2)=1,
$$

and we obtain the difference between the total average 2 and $(3,1)$, two averages of the left and right halves. From this view point, we can see some similarity of this inner product and definitions of Fourier coefficient: in case of $a_{0}$, we compute product of the target data $f(x)$ and 1 for any point of the given range, and then sum up all of the product.

Before stating the sampling theorem, we review sampling. Given a fixed range $\left[x_{1}, x_{n}\right]$, sampling is to choose a finite number of $\left(x_{1}, x_{2}, \cdots, x_{n}\right)$ from the range. Now we assume that $x_{i}-x_{i-1}$ is constant and the $\mathrm{x}$-axis of data is time, that is, data is something changing as time advances. The number of sampling points in a second is called a sampling frequency or sampling rate. So, for example, when we sample two points in a second, the sampling frequency is $2 \mathrm{~Hz}$. 
Now we are ready to explain the sampling theorem. Given a data, let $f_{0}$ to be the lowest frequency included in the data. Then the sampling theorem assures that we can rebuild the original data from sampled data if the sampling rate is greater than $f_{0} / 2$.

To understand the theorem, consider a simple move, uniform circulate motion with period 1s. That is, a ball is uniformly circulating and we see the ball at the same position every one second. In other words, its frequency is $1 \mathrm{~Hz}$ (see Fig. 8).

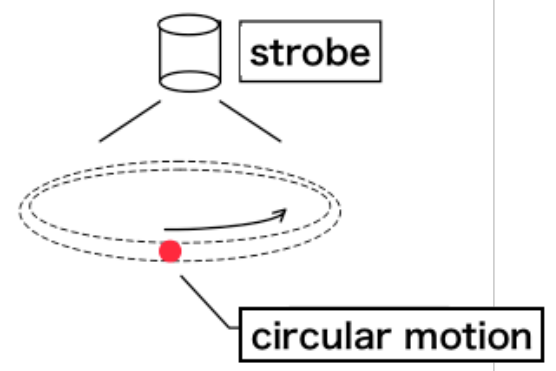

Figure 8: A ball is uniformly circulating in the dark and a strobe is flickering. Under visible light, we can see the ball at any position as it circulates. Therefore, if we consider a function of its positions, then the function is continuous, that is, analog data. When we consider the circulate motion in the dark and we see the ball with flashing light, positions of the ball can be digitized since the two consecutive positions are now discrete, not continuous. When the strobe is flickering at high frequency, we can see the ball at almost any position. For example, let the frequency $f$ be 100 , we can see at 100 positions on its circular movement. With high frequency, we can see almost the same as the original move. In fact, a standard movie camera samples at a few dozen $\mathrm{Hz}$, such as $30 \mathrm{~Hz}$.

Now we consider the following question: how fast frequency is necessary to rebuild the original motion. The sampling theorem tells that we need a high frequency more than $f_{0} / 2$. In the above example, it is necessary a frequency more than $0.5 \mathrm{~Hz}$.

In case of water pearl, we also have two types frequencies (see Fig. 9): one is frequency included in original data and the other is frequency of sampling, that is, timing of flashing light. 

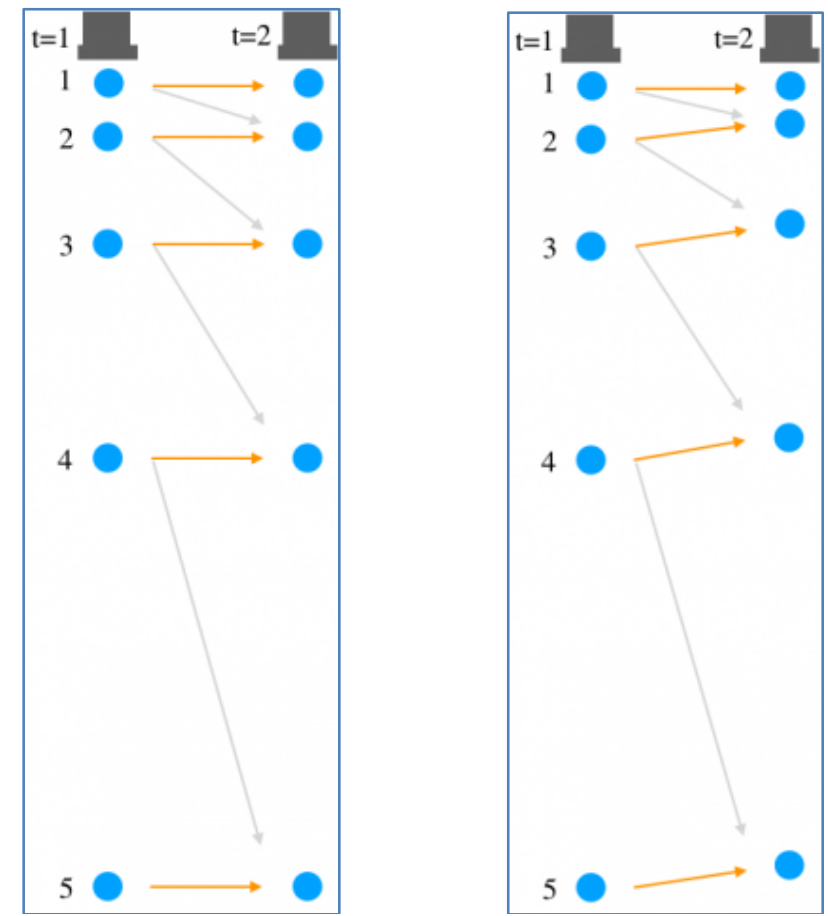

Figure 9: Mechanism of water pearl.

The target data is positions of droplets and sampling is achieved by flashing light in the dark. Now we assume that we drop a droplet every second in the dark and see droplets using flashing light with frequency of $1 \mathrm{~Hz}$ (the left-hand side of the figure). In this case, droplets seem to stay the same positions (orange arrows in the figure) while real movements are shown by gray arrows. Next we choose the timing of the strobe scope to flash every 0.9 second. Such a frequency makes an illusion that droplets are climbing up (orange arrows) while real movements are shown by gray arrows.

\section{ACKNOWLEDGEMENTS}

This work was supported by JSPS KAKENHI Grant Number $15 \mathrm{H} 02787$.

\section{REFERENCES}

[1] T. Bell, I. H. Witten, and M. Fellows. CS Unplugged: An enrichment and extension programme for primary-aged students", Computer Science Unplugged, v3.0 edition, 2015.

[2] T. J. Cortina. "Reaching a Broader Population of Students through 'Unplugged' Activities", Communications of the ACM, 58(3):25-27, 2015.

[3] D. Ikeda, "LESSONS ON INFORMATION IN TERMS OF THE FOURIER TRANSFORM FOR PRIMARY-AGED STUDENTS", Proceedings of the 12th Annual International Technology, Education and Development Conference (INTED2018), pp.3734-3739, 2018. 